\title{
Turbulence in cooling channels of rocket engines: Large Eddy Simulations
}

\author{
Cécile Münch*, Olivier Métais \\ L.E.G.I, BP 53, 38041 Grenoble cedex 09, France
}

Received 24 May 2005; accepted 30 May 2005

Available online 1 July 2005

Presented by Marcel Lesieur

\begin{abstract}
The aim of this Note is to predict by means of large eddy simulations the three-dimensional structures and secondary mass and heat fluxes which develop within a heated curved duct, for applications to rocket engines cooling channels. We show the existence of unsteady Görtler-type vortices above the concave wall, as well as intense secondary vortices taking the shape of two quasi-steady counter-rotating cells of Ekman type close to the convex wall. These cells control heat exchanges. They induce ejections and sweeps close to the convex wall when it is heated. In this case the Nusselt number undergoes strong transverse fluctuations which might induce material alterations. To cite this article: C. Münch, O. Métais, C. R. Mecanique 333 (2005). (C) 2005 Académie des sciences. Published by Elsevier SAS. All rights reserved.
\end{abstract}

\section{Résumé}

Turbulence dans les canaux de refroidissement des moteurs de fusée : Simulation des Grandes Echelles. L'objectif de cette Note est de prédire par simulation des grandes échelles les structures tridimensionnelles et les flux secondaires de masse et de chaleur se développant dans un conduit courbe chauffé, pour des applications aux canaux de refroidissement des moteurs de fusée. On montre l'existence de tourbillons instationnaires de type Görtler sur la paroi concave, ainsi que des tourbillons secondaires intenses formant deux cellules contra-rotatives quasi-stationnaires de type Ekman près de la paroi convexe. Ces cellules contrôlent les échanges thermiques. Elles induisent des mécanismes d'ejections et de balayages près de la paroi convexe quand elle est chauffée. Ceci provoque de fortes variations transverses du nombre de Nusselt qui pourraient conduire à une dégradation des matériaux. Pour citer cet article: C. Münch, O. Métais, C. R. Mecanique 333 (2005).

(c) 2005 Académie des sciences. Published by Elsevier SAS. All rights reserved.

Keywords: Turbulence; Heat transfer; Computational fluid mechanics

Mots-clés : Turbulence; Transferts thermiques; Mécanique des fluides numérique

\footnotetext{
* Corresponding author.

E-mail addresses: cecile.munch@hmg.inpg.fr (C. Münch), olivier.metais@hmg.inpg.fr (O. Métais).
} 


\section{Introduction}

The prediction of heat and mass transport processes in curved ducts is of interest for engineering applications like compressors, turbines or cooling ducts of rocket engines. Several experimental investigations have been performed to study the turbulent flow within curved ducts without any heating: [1,2]. These works have brought to light the destabilizing effect of the concave wall when the convex wall has conversely a stabilizing action. Resulting from this centrifugal instability, Görtler vortices appear on the concave wall and an intense cross-stream flow develops. Numerical studies have been performed by [2,3]: the difficulty lies in the correct prediction of this crossstream flow (called secondary flow) and of the related turbulence characteristics. As far as the relationship between Görtler vortices and heat transfer is concerned, Toe et al. [4] have experimentally investigated the boundary layer over a concave heated wall of a duct. No numerical work has yet been devoted to the study of the combined effect of curvature and heating in a closed duct for a turbulent regime. We here perform Large Eddy Simulation (LES) to investigate the turbulent flow and the associated coherent vortices and structures in a curved square duct. After having presented the numerical procedure (Section 2), we first study the curved duct without any heating (referred to as non-heated duct). Then we consider two distinct configurations: heating applied respectively on the concave and convex walls. Our main goal is to investigate the mutual influence of the secondary flow and the heat transfer.

\section{Numerical methods}

We solved the LES modified three-dimensional compressible Navier-Stokes equations (see [5]). The subgridscale model is the selective structure-function model proposed by [6]. Three supplementary relations and equations are considered: (i) the Sutherland empirical law to describe the molecular-viscosity variation with temperature; (ii) the gas is considered as an ideal gas with the corresponding equation of state; (iii) the turbulent Prandtl number is equal to 0.6. Curvilinear coordinates are used and the equations are discretized with a fully-explicit McCormack scheme [7], second-order in time and fourth-order in space. On Fig. 1(a), the computational-domain geometry is presented. The curved duct has a length of $13.6 D_{h}$ in the streamwise direction $(s), D_{h}$ being the hydraulic diameter. The parameters of the simulations are the following: Reynolds number, $R e=6000$ based on the mean bulk velocity $U_{b}$ (see [8] for definition); Mach number $M a=0.5$; molecular Prandtl number $\operatorname{Pr}=0.7$. A non-uniform numerical grid is used with $160 \times 50 \times 50$ nodes, in the streamwise direction (called $s$ ), the direction normal to the curved walls (called $n$ ) and the spanwise direction (called $z$ ) respectively. In the $n$ and $z$ directions, an hyperbolic-tangent stretching is utilized: the first node close to the wall is located 1.8 wall units away. We here use the same grid resolution as [8] for the straight duct with the same Reynolds number; indeed, these authors have shown that such a resolution is sufficient to obtain good comparisons with DNS and laboratory experiments. The boundary conditions on the four walls consist in a no-slip velocity assumption, and a uniform temperature. One original feature of the

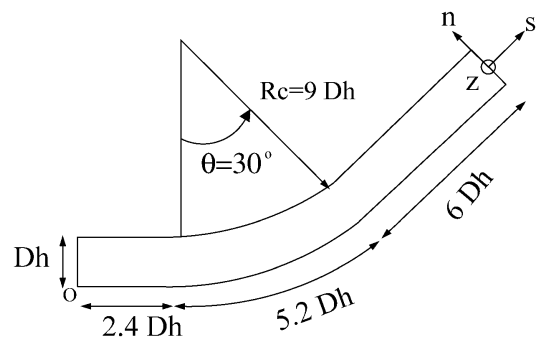

(a)

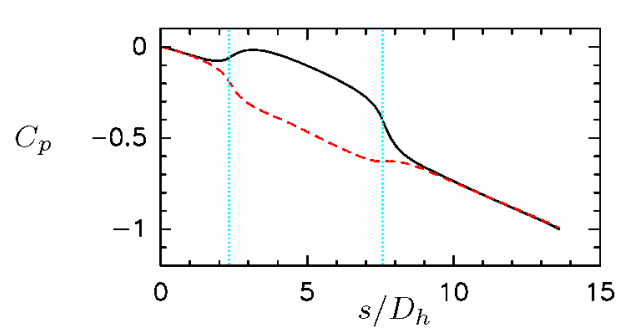

(b)

Fig. 1. (a) Geometry of the computational domain; (b) Pressure coefficient on the curved wall - concave, -- - convex.

Fig. 1. (a) Géométrie du domaine de calcul; (b) Coefficient de pression sur la paroi — concave, - - - convexe. 
present computation is that a fully-developed turbulent state is achieved at the duct inlet thanks to the simultaneous computation of a periodic duct which provides a turbulent inflow field at each instant. The inflow and outflow boundary conditions are obtained through the use of the characteristics conditions proposed by [9].

\section{Non-heated ducts}

We first consider a duct with all its walls at the same imposed temperature $T_{w}$. On Fig. 1(b), we represent on both curved walls the non-dimensionalized pressure coefficient $C_{p}$, defined by $C_{p}=\left(p-p_{i}\right) /\left(p_{o}-p_{i}\right)$, where $p$ is the pressure at the wall. $p_{o}$ and $p_{i}$ are the pressure at the outflow and the inflow respectively. The vertical lines denote the start and the end of the curved part. Just before the beginning of the curvature, we observe that $C_{p}$ rises on the concave wall and decreases on the convex side. Then, in the curved part, $C_{p}$ declines on both walls, but the difference between the two curves remains steady. After the end of the curvature, the two $C_{p}$ becomes identical on both walls showing the disappearance of the radial pressure gradient. This radial pressure gradient is obviously created to balance the centrifugal forces, as pointed out by [1], and generates strong secondary flows. On Fig. 2, we display the mean velocity projected on sections perpendicular to the mean flow, in order to display this secondary flow. Note that the mean quantities are determined through time averaging. In the duct inlet, confinement-induced counter-rotating vortices develop, two in each corner, as found by [8]. From the beginning of the curved part, the centrifugal force associated with the azimuthal velocity component $U$ is at hand. The centrifugal force is maximum near the duct core where $U$ is maximum, while it becomes zero near the wall due to the vanishing velocity. The fluid is therefore pushed towards the concave wall near the duct center: due to mass conservation, it leads to an intense stream flowing from the concave towards the convex wall near the side walls as shown on Fig. 2(b). Once this stream has impacted the convex wall, the accumulation of fluid leads, near the duct symmetry plane, to a fluid ejection away from the convex wall. Eventually, this transverse flow generates two rotating cells near the convex wall (see Figs. 2(c) and 2(d)). As we move downstream, these two cells of Ekman's type come closer intensifying the strong ejection in the middle plane of the convex wall. Note that two extra vortices of small size form in the duct corners of the convex wall: these are of opposite sign with respect to the two main vortices. On Fig. 3(a), we represent the maximum value in each cross section of the secondary flow intensity defined as $\max _{n, z}\left(\sqrt{\left(V / U_{b}\right)^{2}+\left(W / U_{b}\right)^{2}}\right)$ as a function of the downstream distance in the duct. In the inlet part, the maximum intensity reaches about $2 \%$ of the bulk velocity: this is consistent with the results found by [8] in the straight duct. Right from the beginning of the curved part (first dotted line) the cross flow intensifies reaching around $24 \%$ of the bulk velocity near the end

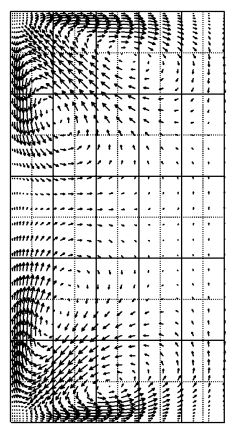

(a)

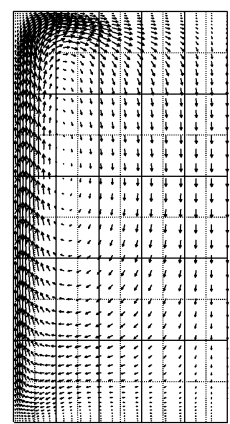

(b)

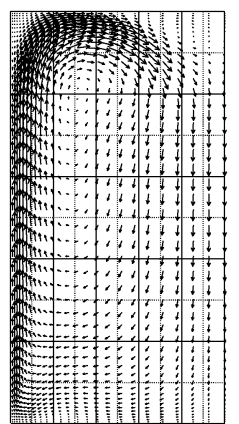

(c)

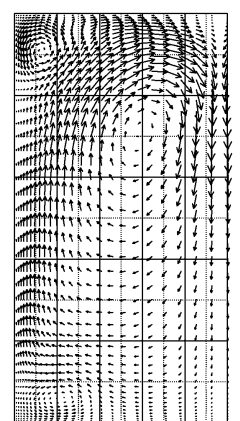

(d)

Fig. 2. Mean velocity vectors projected on cross-sections of the curved isothermal duct showing the secondary flows for: (a) $s / D_{h}=0.5$; (b) $s / D_{h}=5$; (c) $s / D_{h}=7.6$; (d) $s / D_{h}=13.6$. The concave wall is at the bottom of each section.

Fig. 2. Flux secondaires moyens dans des sections du conduit non chauffé en : (a) $s / D_{h}=0,5$; (b) $s / D_{h}=5$; (c) $s / D_{h}=7,6$; (d) $s / D_{h}=13,6$. La paroi concave se situe au bas de chaque section. 


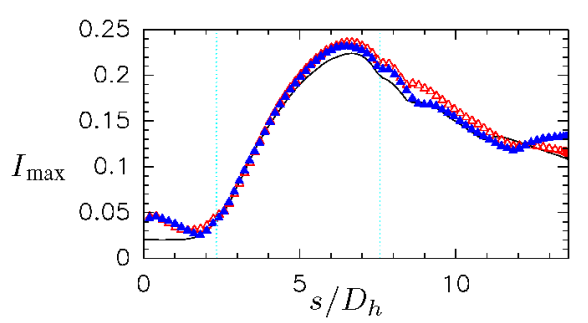

(a)

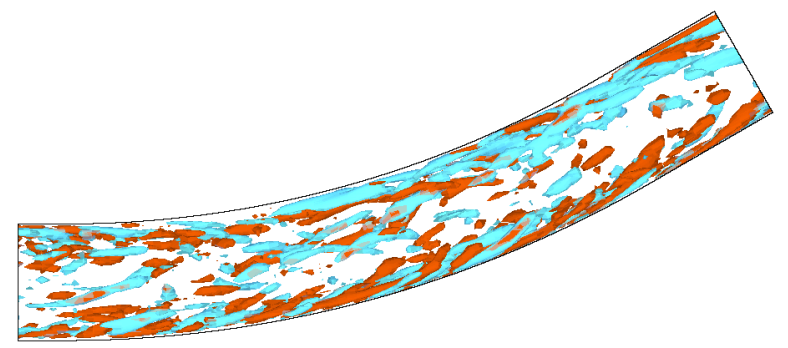

(b)

Fig. 3. (a) Maximum of the mean secondary flow intensity: - non-heated duct, $\triangle$ : heated concave wall, $\mathbf{\Delta}$ : heated convex wall; (b) visualization of the Görtler vortices through $Q$-isosurfaces $=0.6 U_{b}^{2} / D_{h}^{2}$.

Fig. 3. (a) Intensité maximale des flux secondaires : — sans chauffage, $\triangle$ : paroi concave chauffée, $\boldsymbol{\Lambda}$ : paroi convexe chauffée ; (b) visualisation des tourbillons de Görtler par iso-surfaces de $Q=0,6 U_{b}^{2} / D_{h}^{2}$.

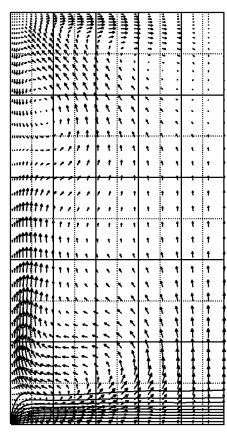

(a)

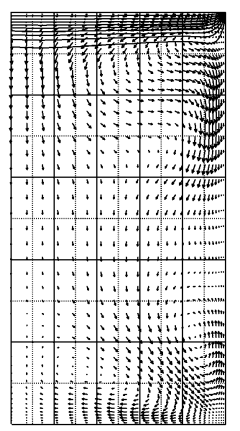

(b)

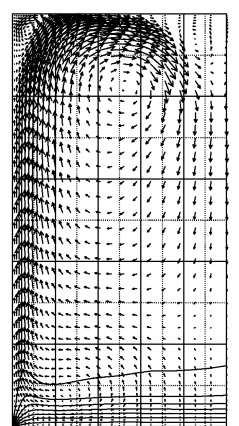

(c)

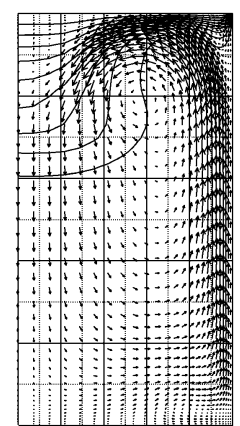

(d)

Fig. 4. Mean velocity vectors projected on two cross-sections of both curved heated ducts, and isolines of mean temperature (step [0.3]): at $s / D_{h}=1$ : (a) concave, (b) convex heated wall; at $s / D_{h}=8.7$ : (c) concave, (d) convex heated wall.

Fig. 4. Flux secondaires dans les deux conduits chauffés et iso-valeurs de la température (pas [0,3]) : en $s / D_{h}=1:$ paroi (a) concave chauffée, (b) convexe chauffée; en $s / D_{h}=8,7$ : paroi (c) concave chauffée, (d) convexe chauffée.

of the curved part. The straight outlet part induces a net decrease but the intensity is still much stronger than at the inlet.

Another aspect of this complex flow is the development of Görtler vortices on the concave wall due to the centrifugal instability. These three-dimensional unsteady structures can be identified through $Q$ isosurfaces. $Q$, the second invariant of the velocity-gradient tensor, constitutes a good way to identify intense vortices [10]. We display $Q$-isosurfaces colored by the longitudinal vorticity in the curved part of the duct on Fig. 3(b). Flow animations show that the Görtler vortices originally forming on the concave wall are driven towards the convex wall by the pressure gradient. They are subsequently trapped in the core of the Ekman vortices where they become quasi-steady.

\section{Heated curved ducts}

We now discuss the heat transfers in a curved duct with a differential heating between the internal and outer boundaries. The gravitational effects are here negligible and all the changes are due to compressibility. We simulate two distinct cases to study the influence of the two different types of curvature on heat transfer. Firstly, the temperature of the concave wall is taken equal to three times the temperature of the other three walls, $T_{w}$. Secondly, heating is applied in the same manner but on the convex wall. In Fig. 4, we represent the secondary flows and the 


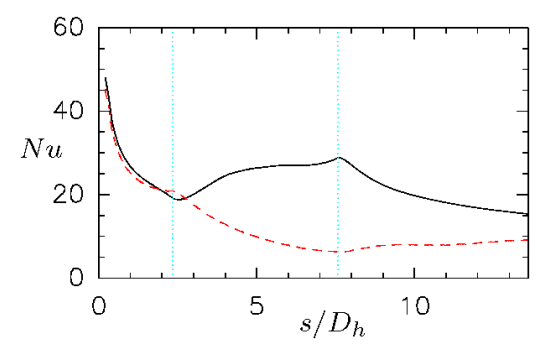

(a)

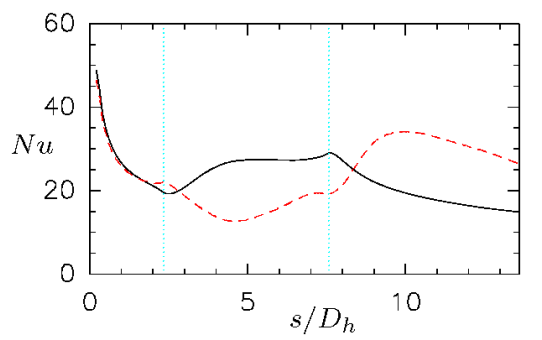

(b)

Fig. 5. (a) Nusselt number in the duct central plane, $z / D_{h}=0.5$; (b) in the plane $z / D_{h}=0.25$ in the curved duct with heating applied on the concave wall -, on the convex wall ---.

Fig. 5. (a) Nombre de Nusselt dans le plan de symétrie, $z / D_{h}=0,5$; (b) dans le plan $z / D_{h}=0,25$ dans le conduit courbe chauffé sur la paroi concave - , convexe ---.

isolines of mean temperature for both cases of heating. From the beginning of the duct, we observe a significant modification of the secondary flow. Indeed, its intensity becomes higher not only close to the heated wall but also on the sidewalls. In both cases of heating, according to Fig. 3(a), the maximum of intensity reaches values up to 5\% of the bulk velocity at the inflow. Within the curved part, at $s / D_{h}=8.7$, curvature effects become dominant. We may, however, observe some significant modifications induced by the heating. When the convex wall is heated, the two Ekman vortices recede from each other and from the convex wall. Moreover the secondary flow maximum intensity is slightly higher than in the non-heated case (see Fig. 3(a)). We next investigate the mutual influence of the secondary vortices on the heat transfer. On Figs. 4(a) and 4(b), temperature isolines are very similar in both cases near the heated wall. After the curvature, the existence of the Ekman cells close to the convex wall leads to a very distinct development of the thermal boundary layer in both cases. When the convex wall is heated, the secondary flow drives hot fluid deep in the core region. Conversely, for concave wall heating, the thermal boundary layer thickness is quasi-homogeneous. Another way to quantify heat exchanges consists in displaying the Nusselt number defined by: $N u(s, z)=\left.k(T) \frac{\partial T(s, n, z)}{\partial n}\right|_{\text {wall }} /\left(\frac{k\left(T_{w}\right) T_{w}}{D_{h}}\right)$, where $k(T)$ is the fluid thermal conductivity. We have plotted $N u$ as a function of $s / D_{h}$ in the symmetry plane and closer to the sidewalls, at $z / D_{h}=0.25$ on Figs. 5(a) and 5(b). At the inflow, the thermal boundary layer development is similar for the two cases of heating and thus $N u(s)$ decreases at the same rate. Conversely, the behaviour of $N u$ is notably different in the curved part depending on the nature of the heating. When the convex wall is heated, the strong ejection of hot fluid away from the heated wall due to the formation of the Ekman vortices induces a progressive decrease of the temperature gradients normal to the heated wall and therefore a continual decrease of the heat flux in the duct central plane. Conversely, when the concave wall is heated, the early decrease in the straight part is followed by a strong increase. This second stage is attributable to the formation of the Ekman cells which induce an intense transport of cold fluid away from the convex wall towards the concave hot wall: this amplifies the temperature gradients near the concave wall and consequently the heat flux. At the end of the curved part, the difference between the two fluxes reaches its maximum: concave wall heating yields a heat flux five times higher than convex wall heating. The strong relationship between the Ekman vortices and the heat flux is confirmed by the evolution of $N u$ in the straight outlet part of the duct. For convex wall heating, the decrease of the Ekman cells intensity is associated with a heat-flux increase. The opposite takes place for concave wall heating with a diminution of the heat flux. In the plane $z / D_{h}=0.25$, $\mathrm{Nu}$ behaves similarly to the central plane for concave wall heating. It is very different when the heating is applied on the convex wall: the Ekman cells indeed drive cold fluid from the sidewalls toward the convex wall. This sweep of cold fluid impacting the heated wall plays a significant role near $z / D_{h}=0.25$ and creates a rise of the Nusselt number (Fig. 5(b)) which proceeds even after the end of the curved part. The subsequent decrease can be explained by the displacement of the Ekman cells towards to duct center and by the decrease of the Ekman cells intensity. 


\section{Conclusion}

LES of the turbulent flow in curved square duct have been carried out to investigate curvature effects. We have observed that an intense secondary motion develops perpendicular to the mean flow, due to the lack of balance between the centrifugal force and the radial pressure gradient. Two quasi-steady counter rotating cells of Ekman type appear near the convex wall and Görtler vortices develop on the concave side. We have next investigated the combined effects of curvature and heating and separately considered the case of the heating applied on the duct concave wall or on the convex wall. For concave wall heating, the heat flux is enhanced due to the presence of the Ekman cells near the opposite convex wall which transport cold fluid towards the heated wall. For convex wall heating, the Ekman cells generate an ejection of hot fluid in the symmetry plane region associated with a weak heat flux. The sweeping motion of cold fluid towards the heated wall on each side of the central ejection is conversely linked with an enhancement of the heat flux. These combined sweeping and ejection motions induce a strong transverse variations of the Nusselt number. In practical situations such as the cooling channels of rocket engines, it can yield important transverse thermal constraints and significant material alterations of the heated convex wall.

\section{Acknowledgements}

Some of the computations were carried out at the IDRIS (Institut du Développement et des Ressources en Informatique Scientifique, Paris) and supported by the CNES (Centre National d'Etudes Spatiales).

\section{References}

[1] S.M. Chang, J.A.C. Humphrey, A. Modavi, Turbulent flow in a strongly curved U-bend and downstream tangent of square cross-sections, Int. J. PhysicoChemical Hydrodynamics 4 (3) (1983) 243-269.

[2] W.J. Kim, V.C. Patel, Origin and decay of longitudinal vortices in developing flow in a curved rectangular duct, J. Fluids Engrg. 116 (1994) 45-52.

[3] A. Silva Lopes, U. Piomelli, J.M.L.M. Palma, Large eddy simulation of the flow in an S-duct, AIAA 0964, 2003.

[4] R. Toe, A. Ajakh, H. Peerhossaini, Heat transfer enhancement by Görtler instability, Int. J. Heat Fluid Flow 23 (2002) $194-204$.

[5] M. Lesieur, P. Comte, Filtrage de Favre et macro-température en simulation des grande séchelles de la turbulence compressible, C. R. Acad. Sci. Paris Ser. IIb 329 (2001) 363-368.

[6] M. Lesieur, O. Métais, New trends in large eddy simulations of turbulence, Ann. Rev. Fluid Mech. 28 (1996) 45-82.

[7] C.A. Kennedy, M.H. Carpenter, Comparison of several numerical Methods for simulation of compressible shear layers, NASA technical paper, Paper 3484, 1997.

[8] M. Salinas Vazquez, O. Metais, Large-eddy simulation of the turbulent flow through a heated square duct, J. Fluid Mech. 453 (2002) 201-238.

[9] T. Poinsot, S. Lele, Boundary conditions for direct simulations of compressible viscous flows, J. Comput. Phys. 101 (1992) $104-129$.

[10] J. Hunt, A. Wray, P. Moin, Eddies, stream, and convergence zones in turbulent flows, Center of Turbulence Research, CTR-S88, 1988. 